\title{
EchoGéo
}

19 | 2012

Du littoral à la haute mer : quelles recherches récentes en géographie?

\section{Le détroit de Gibraltar dans la mondialisation des transports maritimes}

Capter les circulations marchandes pour créer du développement

\section{Nora Mareï}

\section{(2) OpenEdition}

\section{Journals}

\section{Édition électronique}

URL : https://journals.openedition.org/echogeo/12919

DOI : 10.4000/echogeo.12919

ISSN : 1963-1197

Éditeur

Pôle de recherche pour l'organisation et la diffusion de l'information géographique (CNRS UMR 8586)

\section{Référence électronique}

Nora Mareï, «Le détroit de Gibraltar dans la mondialisation des transports maritimes », EchoGéo [En ligne], 19 | 2012, mis en ligne le 10 février 2012, consulté le 21 septembre 2021. URL : http:// journals.openedition.org/echogeo/12919; DOI : https://doi.org/10.4000/echogeo.12919

Ce document a été généré automatiquement le 21 septembre 2021.

EchoGéo est mis à disposition selon les termes de la licence Creative Commons Attribution - Pas d'Utilisation Commerciale - Pas de Modification 4.0 International (CC BY-NC-ND) 


\title{
Le détroit de Gibraltar dans la mondialisation des transports maritimes
}

Capter les circulations marchandes pour créer du développement

\author{
Nora Mareï
}

Navires au mouillage face à l'enclave britannique de Gibraltar

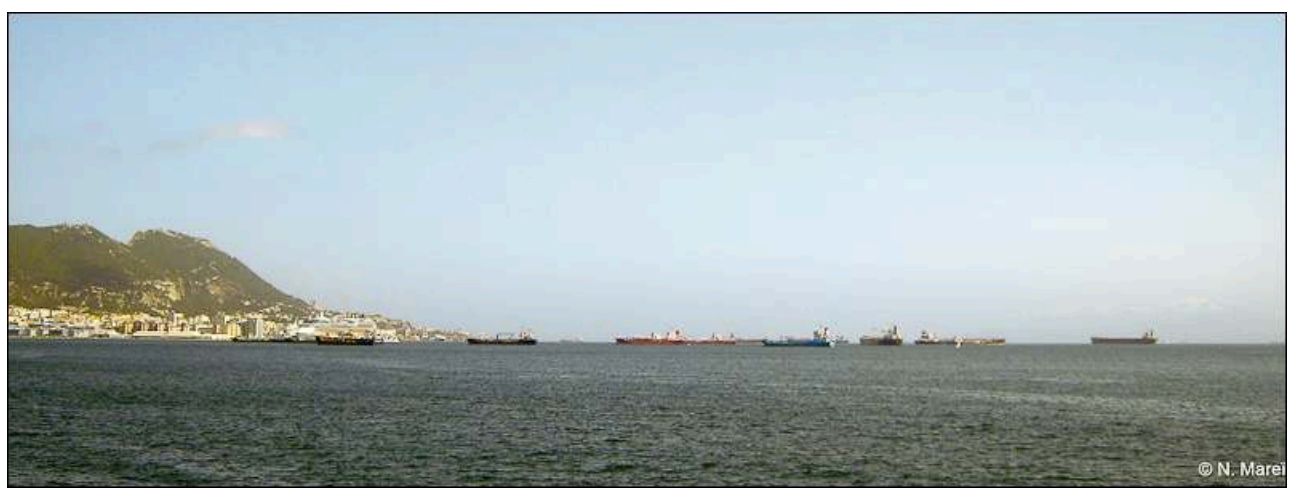

Nora Mareï, juillet 2010.

Depuis toujours, le détroit de Gibraltar est un espace observé et convoité, dont les fonctions ont évolué à chaque époque de l'histoire. L'ouverture du canal de Suez, en 1869, a cependant accéléré son destin. Il devient le goulet majeur des routes maritimes en provenance d'Asie. La grande route impériale des Indes, celle du pétrole arabopersique, ou encore celle des produits manufacturés asiatiques passent systématiquement par Suez et donc Gibraltar pour les échanges entre l'Europe et l'Asie (Charlier, 1990 ; Guillaume, 1999 ; Marcadon, 2004). Il n'est alors pas nouveau de parler de mondialisation en ce qui concerne le détroit de Gibraltar. Toutefois, son rôle évolue rapidement depuis la fin des années 1980 . Il a ainsi été placé sur l'échiquier maritimoportuaire contemporain par l'amateur danois Maersk. Ce dernier, à l'origine de changements structuraux dans les logiques du transport maritime conteneurisé, a 
choisi comme port pivot à l'échelle mondiale le port d'Algeciras sur le détroit de Gibraltar. Il place ainsi le détroit au cœur des restructurations du transport maritime mondial et en fait un observatoire des changements (Vigarié, 1995 ; Guillaume, 2005 ; Frémont, 2007).

2 Malgré ce rôle grandissant sur la scène maritime mondiale, les régions frontalières du détroit de Gibraltar, l'Andalousie d'un côté, le Tangérois de l'autre, sont des régions pauvres et à la périphérie des centres économiques de leur pays respectif. Par l'émergence d'une dynamique portuaire conquérante, elles ont pourtant réussi à s'accrocher aux flux mondiaux passant quotidiennement à leurs pieds. Dans quelle mesure, cet arrimage aux grands flux maritimes circumterrestres influence-t-il le développement des territoires de rivage?

En proposant un regard global sur cet espace terraqué, l'objectif de l'article est d'analyser les enjeux territoriaux d'un espace qui semble plus animé par la mer qui se trouve en son centre que par ses rivages. La première partie évoque la manière dont ce passage maritime international est devenu une plaque tournante de la mondialisation des échanges. Ensuite, un retour à une échelle plus locale permet de révéler les risques de ruptures spatiales engendrés par cet arrimage mondial. Enfin, dans une réflexion sur les solutions à apporter à cette désarticulation entre un système mondialisé et des marges qui peinent à en profiter, la perspective d'une gestion commune des rives du détroit est envisagée.

\section{Illustration 1 - Localisation des ports du détroit et des activités péri-portuaires}

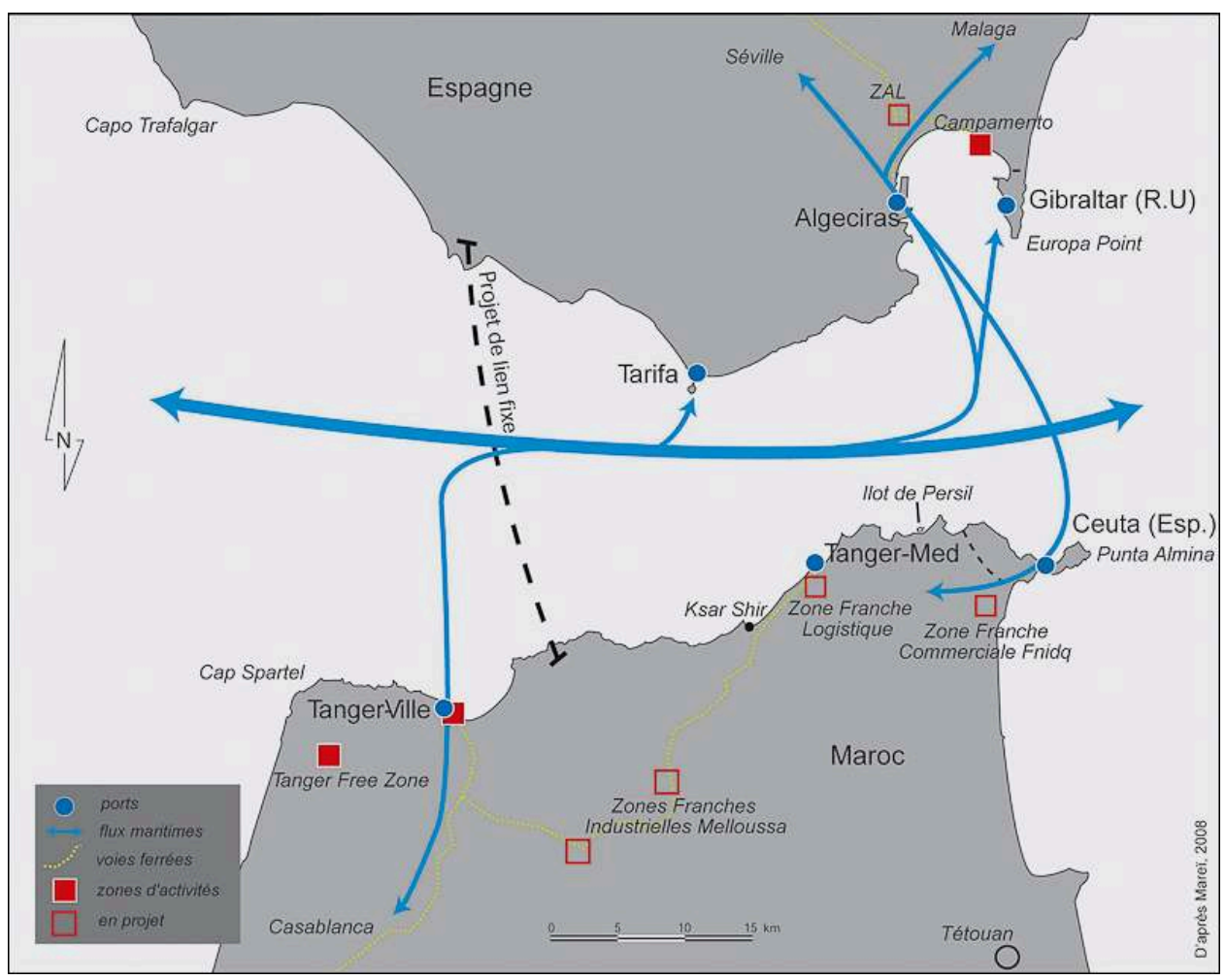

Source : Nora Mareï, 2008. 


\section{Le détroit de Gibraltar, une plaque tournante pour la mondialisation des échanges}

4 Sans être à la tête d'une zone de production internationale ou de consommation de masse, il est rare qu'un espace maritime devienne un lieu d'escale pour les grands navires de la flotte mondiale. Pourtant sur le détroit de Gibraltar, la spécialisation dans le stockage et la redistribution des conteneurs à l'échelle internationale, associée à une forte activité de ravitaillement des navires, ne sont pas loin d'en faire une aire d'arrêt incontournable sur la grande autoroute maritime Est-Ouest. Le détroit de Gibraltar devient un espace original où se croisent le dense trafic marchand alimentant les échanges entre Europe et Asie, et une circulation humaine de plus en plus intense, révélatrice des liens historiques entre l'Europe et l'Afrique. L'ensemble de ces flux hisse le détroit de Gibraltar au rang des détroits du Pas de Calais ou de Malacca en termes de trafic maritime international (97000 à 100000 navires par an) et la nature de ces flux en fait un espace observatoire de la mondialisation des échanges.

\section{Une plate-forme multisite d'éclatement des conteneurs}

5 En à peine dix ans, la situation monopolistique du port d'Algeciras pour les trafics conteneurisés a été bouleversée par l'arrivée d'un concurrent de poids sur l'autre rive, le port marocain de Tanger-Med, et par une situation de saturation des infrastructures du port andalou.

6 Sous l'impulsion du général Franco et de sa politique de développement de pôles industriels en 1965, le petit port d'Algeciras devient un port d'intérêt national. La localisation de ce nouveau pôle industriel est évidente, face à Gibraltar, le gouvernement franquiste étale raffineries, sidérurgies et centrales électriques. Le fond de la baie d'Algeciras devient une véritable zone industrialo-portuaire (ZIP) dont les effets se font sentir encore aujourd'hui puisque une part importante des trafics du port est à imputer aux vracs liquides ( $39 \%)$ et aux vracs secs $(5 \%)$. Pourtant, ce ne sont pas les effets de cette industrialisation lourde qui sont les plus étonnants. Les décisions prises durant les deux décennies suivantes vont bouleverser le sort du port espagnol. En effet, l'arrivée de l'armement Sealand en 1975, puis de Maersk en 1986, vont faire d'Algeciras l'un des premiers ports européens de la conteneurisation. Dès lors, le trafic conteneur ne cesse de croître et le port d'Algeciras, doté en services et infrastructures spécialisées apportés par les opérateurs, s'impose en Espagne, en Europe et en Méditerranée. Exprimé en nombre de conteneurs équivalent vingt pieds (EVP), son trafic le situe au cinquième rang européen en 2007 (près de 3,3 millions d'EVP); il talonne, parfois surpasse, Gioia Tauro à la tête des trafics méditerranéens; et se hisse au premier rang espagnol devant Valence (2,4 millions d'EVP) et Barcelone (près de 2,1 millions d'EVP'). Ce développement portuaire est visible par des extensions spectaculaires de terminaux avançant dans la baie : le terminal Juan Carlos I (69 ha) dédié à Maersk ; et les extensions de Isla Verde Exterior (121 ha), dont les premiers quais ont ouvert durant l'été 2010, et qui ont vu l'arrivée de l'armement coréen Hanjin. Malgré cet imposant déploiement, le port est proche de la saturation.

7 Presque quarante ans plus tard, un scénario semblable se produit sur l'autre rive du détroit. Le Roi du Maroc annonce à l'occasion du discours du trône, le 30 juillet 2002, le lancement d'une opération d'aménagement d'envergure à $40 \mathrm{~km}$ à l'est de Tanger. Il 
s'agit de construire ex nihilo un port en eau profonde, structuré principalement autour de terminaux à conteneurs. Chacun de ces terminaux a trouvé rapidement son concessionnaire, de sorte que les travaux de la première phase, démarrés en 2003, ont permis de faire tourner les deux premiers terminaux en 2007. L'opérateur Maersk, à travers sa filiale APM Terminals y a vu une aubaine afin de consolider sa position sur le détroit. Au regard des 2 millions d'EVP enregistrés en 2010, l'initiative semble couronnée de succès.

\section{Une zone stratégique pour le premier opérateur mondial}

Tanger-Med et Algeciras sont des ports similaires, à classer dans la catégorie des hubs c'est-à-dire des plates-formes d'accueil et de redistribution des conteneurs à une échelle qui peut être régionale ou internationale. Ils sont caractérisés par un taux de transbordement extrêmement élevé : plus de $85 \%$ pour le port d'Algeciras et près de $96 \%$ pour le port de Tanger-Med. Ce taux évoque en quelque sorte le trafic perdu pour l'arrière-pays et permet de les décrire comme de simples lieux de triage des «boîtes » afin de les transférer d'une ligne à l'autre. Pourtant, cette fonction les place au cœur des réseaux maritimes des plus grands opérateurs mondiaux.

Illustration 2 - Réseau secondaire (lignes feeders) de l'armateur Maersk en Méditerranée au départ de ses trois hubs (janvier, 2011)

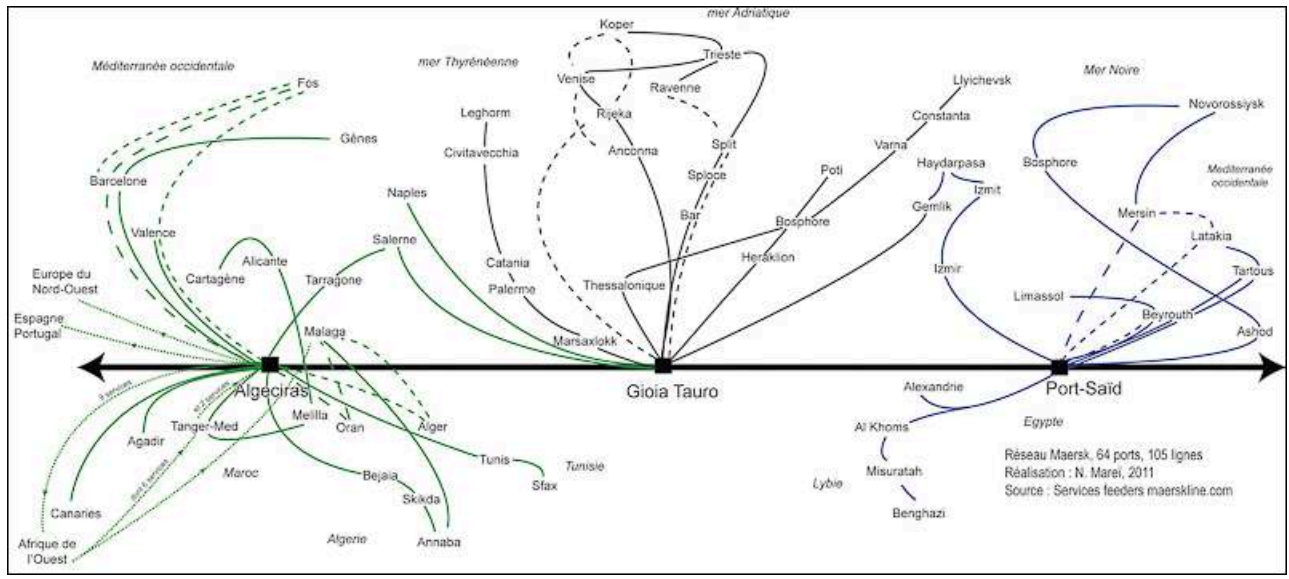

Source : Services feeders maerskline.com ; réalisation : N. Mareï, 2011.

Les stratégies de Maersk en Méditerranée sont révélatrices de l'importance d'un hub dans le réseau maritime d'un transporteur conteneurisé. Le premier opérateur mondial a incontestablement un avantage en Méditerranée par un investissement sur trois passages maritimes : le binôme Algeciras/Tanger-Med sur le détroit de Gibraltar, Gioia Tauro sur le détroit de Messine et Port-Saïd sur le canal de Suez. Ce système permet une couverture complète de la zone méditerranéenne grâce à la mise en place d'un système en hub and spokes. En effet, les hubs sont placés au plus près de la grande route maritime Est-Ouest, la localisation sur les passages internationaux est alors idéale. A partir d'un hub la multiplication des liaisons en arborescences (feeders) permet de multiplier les destinations finales (illustration 2). Cette logique réticulaire permet aux porte-conteneurs géants d'éviter de dévier de l'axe Est-Ouest et donc d'effectuer des rotations plus importantes sur cet axe, tout en augmentant la zone de desserte grâce au réseau secondaire. Cette pratique du hub and spokes, légitiméepar les économies 
d'échelle qu'elle permet et les succès d'opérateur comme Maersk, marque un changement d'époque dans le processus de restructuration du transport maritime mondial et dans la fonction portuaire. Ces activités deviennent de plus en plus enchaînées aux logiques de la logistique internationale (Ducruet, 2005 ; Frémont, 1996).

\section{Une station de service de la mondialisation}

10 Aux croisées des routes maritimes internationales, le détroit de Gibraltar est devenu la première zone méditerranéenne pour l'approvisionnement des navires en fuel (IFO), gas-oil (MGO) et huile marine (MDO). D'une manière générale, de nombreux ports proposent ce genre de services, mais le soutage ou bunkering à gros volumes n'est possible que dans une poignée de zones présentant une situation géographique à proximité des grandes routes maritimes, des installations nécessaires d'accueil des navires et des services complémentaires comme la réparation navale et l'approvisionnement en eaux et en vivres (Castillo Manzano, 2001).

11 Les ports du détroit ont livré 7,96 Mt de soutes en 2007 : 4,3 par Gibraltar ; 3,1 par Algeciras dont la moitié est destinée aux navires de Maersk qui fréquentent le terminal Juan Carlos I ; 560000 t par Ceuta ; et les capacités de soutage de Tanger-Med sont à venir. Ces chiffes restent modestes comparés aux grandes stations de soutage que sont Singapour (32 Mt en 2007), Fujairah dans le golfe Persique (16 Mt) ou Rotterdam (13,6 $\mathrm{Mt})^{2}$, même si l'activité a connu un essor considérable puisqu'en 1990 seulement 800000 tonnes de soutes étaient livrées par les ports du détroit. L'activité est favorisée par la possibilité de soutage sans contraintes de tirant d'eau et les opérations peuvent être réalisées à quai ou en mer par une flotte locale de petits tankers. Le bunkering est néanmoins extrêmement décrié par les associations de protection de l'environnement au nom des risques qu'il engendre pour l'environnement côtier et marin du détroit.

\section{Un lieu incontournable de la traversée Europe-Afrique}

12 L'intense flux marchand de l'artère maritime Est-Ouest est entrecoupé par un trafic Nord-Sud qui n'a cessé de prendre de l'ampleur au cours du XX $\mathrm{X}^{\mathrm{e}}$ s. Il s'agit d'une part, d'un courant marchand support d'une partie du commerce extérieur marocain avec l'Europe et d'autre part, d'un flux humain de plus de 5 millions de passagers dont $40 \%$ traversent le détroit lors de la période estivale ${ }^{3}$ (illustration 3). Les protagonistes de ce grand va-et-vient saisonnier sont en majorité des marocains vivants en Europe qui vont tels "des fourmis", les galeries et remorques de voiture chargées à ras bord, passer un moment dans leur pays d'origine (Tarrius, 1992, Arab, 2009).

13 Au départ de Sète en France, de Gênes ou Livourne en Italie, de Barcelone, Almeria, Malaga et Algeciras en Espagne, ils embarquent à bord d'un ferry à destination du nord du Maroc. En 2011, une flotte de 31 ferries est opérationnelle sur ces lignes. D'un opérateur à l'autre les critères de confort et de modernisme peuvent différer sensiblement. Matthew Murtland (2011) met en exergue la qualité médiocre des ferries marocains. Cette idée est confirmée par l'âge moyen de la flotte des opérateurs marocains du détroit, IMTC et Comarit, dont les navires affichent respectivement des moyennes d'âge de 35 et 31 ans alors que ceux de FRS, Acciona Trasmediterranea et Balearia, les opérateurs espagnols, ont quant à eux des moyennes d'âge de 17, 16 et 10 ans. Ce décalage qui existe entre une flotte vieillissante et un marché en expansion peut 
s'expliquer, entre autres, par le caractère extrêmement saisonnier de cette mobilité massive.

Illustration 3 - Trafics passagers des ports du détroit et de la mer d'Alboran en 2007

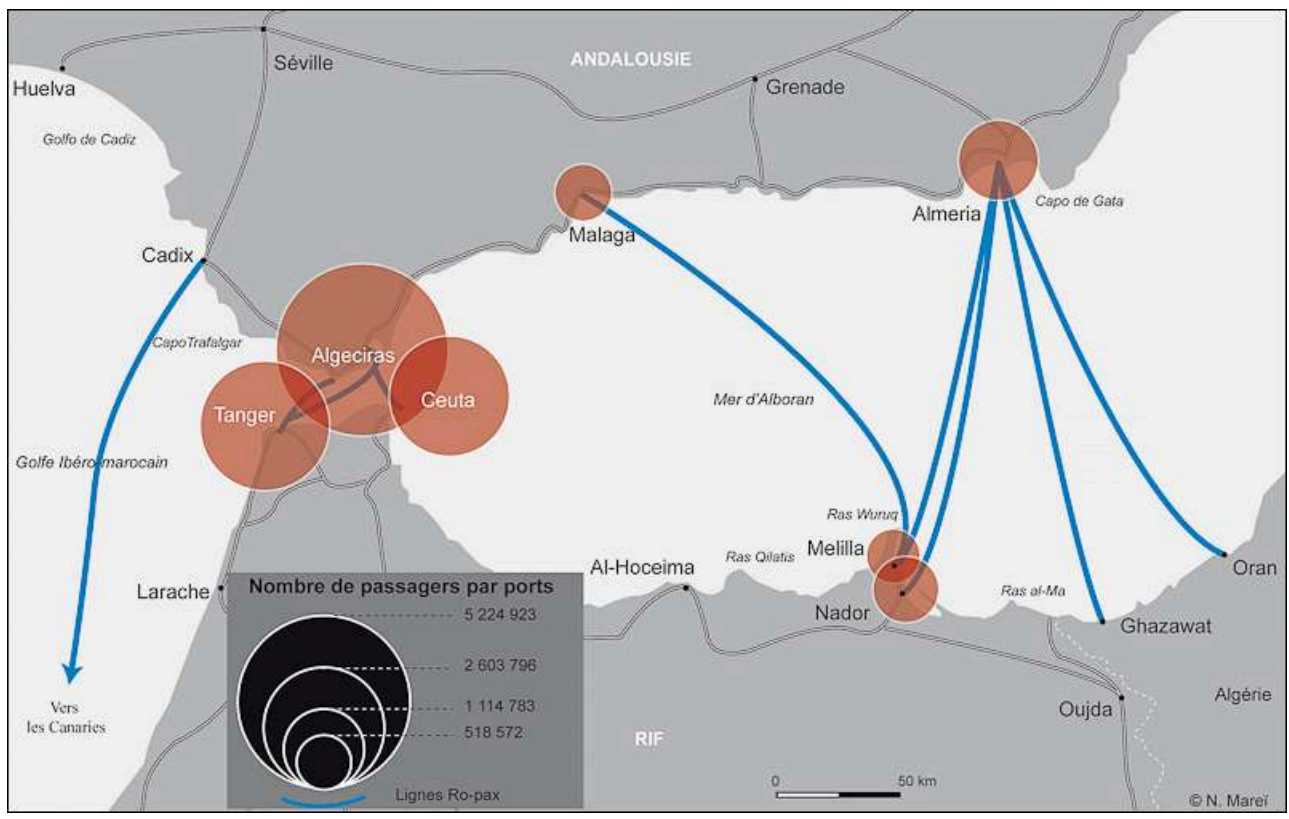

Nora Marei.

\section{Du mondial au local, un espace désarticulé}

Le détroit de Gibraltar est un espace où les circulations humaines et marchandes sont les principales animatrices des territoires. Elles dynamisent les lieux et rythment l'investissement qui consiste au développement d'une nouvelle offre de transport afin d'accueillir des flux en augmentation continue. Par la mise en place de ces infrastructures, principalement portuaires, l'objectif recherché est de s'accrocher aux flux mondiaux; la gestion de ces flux devrait permettre de créer de l'argent et d'impulser du développement. D'une rive à l'autre, les prises de décisions établissent alors l'infrastructure comme l'instrument essentiel du développement territorial projetant ainsi les territoires dans le mythe politique des effets structurants des infrastructures de transport (Offner, 1993). Cependant, la trop forte mise sur un développement capitalistique et économique comporte un risque de dysfonctionnement et de désarticulation spatiale sur un espace littoral sinistré d'un point de vue socio-économique et fragile d'un point de vue environnemental.

\section{La construction d'un espace où les intérêts extraterritoriaux priment}

Opérateurs maritimes et dynamisme portuaire

L'insertion des ports du détroit dans les principaux réseaux conteneurisés de la planète a comme conséquence une appropriation complète du dynamisme portuaire par des intérêts extraterritoriaux, en particulier ceux des plus grands opérateurs mondiaux du transport maritime (Maersk, $1^{\mathrm{er}}$ armateur mondial en 2011; CMA-CGM, $3^{\mathrm{e}}$; Hanjin 
Shipping, $9^{\mathrm{e}}$ ). S'ils participent à la construction des infrastructures portuaires de dernière génération, leur intérêt, en tant que transporteur, est donc essentiellement de développer leur activité vers les avant-pays marins. Il est donc peu probable que ces opérateurs amènent des possibilités importantes d'enracinement territorial et ceci est d'autant plus vrai que le port concerné est un hub. En effet, la valeur ajoutée de la fonction portuaire est très dépendante du type de trafic, et les conteneurs ne sont pas à ranger parmi les trafics les plus intéressants de ce point de vue. En particulier, l'activité de transbordement est en général peu inductrice de valeur ajoutée locale, elle emploie une faible main-d'œuvre et elle peut être très vulnérable en cas de forte concurrence. Les autorités portuaires qui arrivent à bénéficier de belles plus-values sont celles qui ont développé un fort potentiel industriel et logistique péri-portuaire en investissant, par exemple, dans des zones d'activité spécialisées et extrêmement bien connectées à leur arrière-pays. En ce qui concerne l'autorité portuaire d'Algeciras, la prise en compte de ces principes de massification des trafics est très récente et les moyens mis en œuvre pour y arriver sont encore balbutiants.

\section{Tanger-Med, le modèle du développement asiatique importé en Méditerranée}

16 A Tanger Med, la donne est d'emblée différente car le projet est présenté comme une proposition globale d'aménagement de la région du Tangérois. Il est confié à une agence publique créée en 2002, l'Agence Spéciale Tanger Méditerranée (TSMA) dont les compétences sont portuaires mais également terrestres puisqu'elle est désignée comme l'aménageur de la Zone Spéciale de Développement (ZSD) créée pour accompagner le projet. Cependant, comme à Algeciras, la concession des terminaux est dévolue à des sociétés privées (Maersk et CMA-CGM ont remporté les plus importantes concessions), Tanger Med s'inscrivant dans une réforme radicale du système portuaire marocain. Si la concession des terminaux conteneurs reste au cœur du projet, l'originalité est à observer dans la création de cette ZSD qui consiste en la mise en place de zones d'activités intégrées et de zones franches logistique, commerciale et industrielle comme celle de Melloussa ( $600 \mathrm{ha}$ ), orientée vers les activités d'exportation et qui accueillera Renault sur 300 ha en 2012.

Même si le projet a rencontré quelques succès commerciaux grâce à l'arrivée de nouveaux investisseurs au Maroc, le principe même de zones franches interpelle quant à ses effets sur le développement des territoires. En effet, les avantages d'une telle zone sont liés à des législations fiscales extrêmement permissives (pas de droits de douane, pas de TVA, pas d'impôts sur les sociétés pendant cinq ans, rapatriement de capitaux en devises étrangères, etc.). Ce sont des zones qui fonctionnent donc de manière isolée par rapport à leur espace d'accueil. Les spécialistes du bureau international du travail (BIT) reconnaissent la difficulté d'évaluer la contribution de ces zones défiscalisées au développement socio-économique, tout en relevant leurs résultats sur l'emploi, la diversification du tissu économique, et l'attraction des investissements étrangers. Dans cette perspective, l'objectif de faire de la ZSD une zone atelier, place alors les responsables marocains dans une optique complètement différente de celle d'Algeciras. L'appel à l'investissement étranger et la mise en place d'une industrie de main-d'œuvre à vocation exportatrice comme l'assemblage automobile sont alors présentés comme les clefs du développement de la région. De telles entreprises ont connu quelques succès en particulier en Asie (Chine, Singapour, Malaisie). Cependant pour de nombreuses zones de ce genre, il est difficile de sortir du cercle vicieux d'une 
production à faible valeur ajoutée et sans montée en gamme, les effets sur le développement ne vont guère plus loin que la création de quelques emplois sousqualifiés (BIT, 2003 ; Bost, 2007).

18 Ainsi, dans un cas comme dans l'autre, les choix politiques en termes d'aménagements portuaires et péri-portuaires semblent loin de l'enracinement territorial tant souhaité sur ces espaces où les besoins socio-économiques sont énormes. D'ailleurs, de manière assez symptomatique, le commerce extérieur marocain n'est responsable à Tanger Med que de $46228 \mathrm{EVP}$ en 2009, soit moins de $4 \%$ du trafic global du hub.

\section{Une dégradation sensible de l'environnement marin et côtier du détroit}

19 L'intensification de l'activité humaine sur les littoraux peut être source de tensions sur les espaces marins et côtiers environnants, en particulier lorsqu'il s'agit de développer des infrastructures lourdes et dévoreuses d'espace, telles les zones portuaires et industrielles. Un port performant est un port présentant des profondeurs suffisantes afin d'accueillir les plus gros navires de la flotte mondiale et des espaces de stockage conséquents pour héberger, entre autres, les milliers de conteneurs en attente de transbordement ou d'évacuation vers l'arrière-pays. Pour répondre à ces critères physiques de performance, sur les côtes escarpées du détroit de Gibraltar, les ports ont été construits par remblaiement sur les plus grandes baies existantes et par extension de ces remblais en mer. Dans la baie d'Algeciras, 610 hectares ont été gagnés sur la baie en un siècle et le mouvement de remblaiement n'est pas achevé (port d'Algeciras et de Gibraltar). Sur le site de l'Oued R'Mel où a été construit Tanger-Med, la phase $1 \mathrm{du}$ projet à consister à construire une centaine d'hectares de terminaux portuaires intégralement gagnés sur la mer (illustration 4). Ces constructions lourdes sont responsables de perturbations importantes dans le dynamisme morpho-sédimentaire des plages du détroit (érosion) au point que plusieurs campagnes de rechargement en sable des plages de la baie d'Algeciras ont été menées ces dernières années. Il faut ajouter à ces remblaiements strictement côtiers ceux des zones rétro-littorales correspondant aux zones d'activités économiques ou logistiques et qui participent alors à l'imperméabilisation des sols dans des zones soumises à de fortes intempéries hivernales.

Illustration 4 - Evolution diachronique des infrastructures portuaires de Tanger-Med

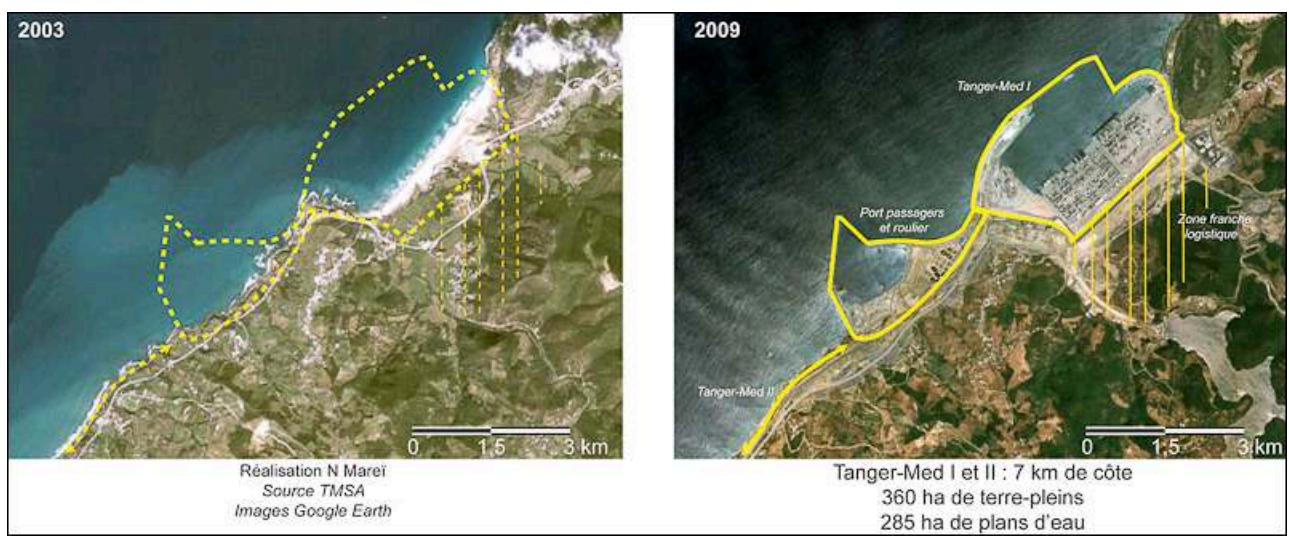

Source : TMSA, images Google Earth ; réalisation : N. Mareï. 
Outre ces déséquilibres les plus visibles, il ne faut pas omettre les risques liés à la fonction de zone de transit et d'escale internationale pour une grande partie de la flotte mondiale. Les accidents maritimes ne sont pas rares dans le détroit et leurs effets sont souvent amplifiés par le mauvais temps et la lenteur d'intervention dans des eaux sans souveraineté officielle. Le 12 août 2007, le vraquier panaméen New Flame a heurté le pétrolier danois Torm Gertrud alors qu'il sortait du port de Gibraltar. À la suite de cette collision, le cargo New Flame s'est échoué face à la pointe de l'Europe (Gibraltar). Pendant six mois, aucune solution n'a été apportée à l'échouage du bateau jusqu'à ce que le 10 février 2008, une tempête provoque une fuite d'hydrocarbures provenant du cargo, polluant ainsi 1,5 km de côtes espagnoles. La même année le Sierra Nava, navire frigorifique battant pavillon panaméen, s'est échoué sur une plage du sud de la baie d'Algeciras et a contaminé aux hydrocarbures 1 kilomètre de côtes. A ces accidents graves, il faut ajouter une pollution manifeste des eaux causée par des dégazages sauvages, les fuites liées à l'activité de soutage et considérées comme fréquentes par les associations écologistes, les déversements industriels de la baie d'Algeciras et l'évacuation directe en mer des eaux usées de l'ensemble des villes du détroit.

21 Tous ces risques marins et côtiers sont retrouvés sur l'ensemble du pourtour méditerranéen où le littoral subit un surinvestissement économique (industrie portuaire, tourisme, villes pluri-millionnaires, etc.). Le cas du détroit de Gibraltar n'est donc pas isolé, il interpelle simplement par la suraccumulation et la fragilité visible d'un littoral récemment classé pour sa richesse écologique.

\section{Une augmentation des effets de fermeture et d'enclavement spatiaux}

Le dernier risque majeur pour les riverains du détroit est une augmentation des « effets frontières ». Ces effets sont dus à l'existence de flux illégaux qui traversent les territoires riverains du détroit de Gibraltar et qui obligent à la création d'une zone «mirador» avec systèmes radar de surveillance de détroit (SIVE), patrouilles renforcées, et militarisation des frontières, afin de prévenir l'immigration clandestine, le trafic de drogue, la contrebande.

La configuration politique et géographique du détroit, bousculée par un jeu d'enclaves stratégiquement positionnées et inlassablement contestées et revendiquées, favorise l'émergence d'un courant d'échange illicite. Il s'explique d'une part par le fonctionnement économique des enclaves de Gibraltar et de Ceuta proche de celui d'un port franc et d'autre part par le va-et-vient régulier de travailleurs vivant dans l'arrière-pays andalou ou rifain. L'existence de marchandises détaxées et la possibilité de les faire traverser la frontière moyennant quelques ruses et la corruption de quelques douaniers, alimentent alors régulièrement un courant de contrebande. Cigarettes et alcool en provenance de Gibraltar sont aisément retrouvés dans les rues andalouses; quant aux Marocains, ils sont à la recherche d'électroménager de tous types en provenance d'Europe.

Ces flux marchands illégaux sont accompagnés d'une mobilité humaine clandestine stoppée par la coupure d'eau à franchir que représente le détroit et par la double barrière barbelée et militarisée de la frontière de Ceuta. Ces obstacles n'arrêtent évidemment pas les candidats au passage vers l'Europe. Ils rôdent également aux entrées du port de Tanger à la recherche d'une faille dans le système de surveillance 
leur permettant alors d'embarquer furtivement dans un navire, sous les essieux d'un camion, dans une remorque, etc. Selon une étude du ministère de l'économie et des finances marocain (2008), $0,5 \%$ à $1 \%$ des chargements sont affectés par ces problèmes de sûreté. La migration de l'activité roulier-passager (ro-pax) vers Tanger-Med, au sein d'un port hors de la ville, aux dernières normes de sécurité, et isolé par la mer d'un côté, la montagne de l'autre, permet d'une certaine manière de remédier à ces difficultés. Pourtant, cette situation présente une part de paradoxe puisque partout dans le monde l'activité ferry est par essence la plus «urbaine» des activités portuaires.

Enfin, d'un point de vue économique, le pari marocain sur les zones franches n'est pas dénué de risques puisqu'il favorise un "mitage défiscalisé » des territoires, sans garantie de résorber les équilibres spatiaux, et avec l'éventualité de créer une parcelle de modernité et de richesse aux intérêts exclusivement extraterritoriaux (Coudroy de Lille, 2007). Emergent alors le long des côtes du détroit divers îlots politiques, économiques, militaires, qui fractionnent de plus en plus des territoires déjà peu articulés.

\section{Le détroit de Gibraltar, un espace pertinent d'intégration territoriale?}

Parler d'intégration territoriale sur un espace si complexe peut paraître un vœu pieux au regard des modalités politiques, socio-économiques et environnementales qui viennent d'être passées en revue. Or c'est une piste à explorer afin d'articuler le réseau mondial Est-Ouest dans lequel est inséré le détroit et le système local Nord-Sud en demande d'opportunités de développement. Ici, en particulier, le contact fragile entre la mondialisation et ses marges est exacerbé au risque de faire du détroit un simple lieu de transit d'hommes et de marchandises. Penser à une intégration régionale d'un genre nouveau puisque intercontinentale et euro-africaine est alors peut-être une nouvelle voie du développement pour les territoires marginaux et frontaliers de l'espace européen.

\section{Les efforts à réaliser en vue d'une intégration régionale}

Un projet de gestion commune d'une zone transfrontalière nécessite, outre une volonté politique forte et partagée, une certaine harmonie sociale, économique et politique entre les participants. Une évolution de ce type sur le détroit de Gibraltar implique alors que la région du Tangérois effectue un véritable rattrapage en terme de développement économique, le projet Tanger-Med se présente comme le reflet des aspirations marocaines. Le Maroc devra également «imposer une réelle politique de régionalisation en octroyant à la région [du Rif ou du Tangerois] une responsabilité financière accrue », car la coopération imaginée n'est pas intergouvernementale mais bien interrégionale entre des acteurs et des territoires partageant les mêmes attaches pour l'espace en question (Cherkaoui, 2006). D'autant plus que l'Andalousie voisine est une Communauté Autonome avec ses propres instances politiques, financières et législatives. Il est possible d'imaginer qu'au sein de cet espace solidaire, les platesformes d'éclatement de Tanger-Med et Algeciras coopéreraient, ce qui offrirait une 
capacité d'accueil de quasi 15 millions de conteneurs pour le détroit, devenant ainsi la première zone conteneurisée de l'espace euro-méditerranéen.

Toutefois, sans solution politique pour Ceuta et Gibraltar, le statut quo risque de s'éterniser. Certains responsables espagnols accusent même le Maroc de vouloir casser l'économie de Ceuta avec le projet Tanger-Med. Pourtant, une cogestion de Ceuta avec le Maroc et de Gibraltar avec le Royaume-Uni est envisageable en raison de l'évolution socioculturelle des enclaves. Dans ces enclaves les brassages ethniques sont pluriséculaires et la proportion des résidents marocains à Ceuta ou espagnols à Gibraltar a considérablement augmenté. Yves Zurlo (2006) parle, concernant Ceuta et Melilla (autre enclave espagnole au Maroc), de laboratoire de convivencia signifiant vie en commun en espagnol. Pourtant, les enclaves semblent évoluer plutôt vers l'autonomie politique que la gestion commune.

\section{Les obstacles à l'intégration}

29 Les obstacles à l'intégration sont encore de taille et ils sont à chercher dans les nombreux paradoxes qui émergent de la trop grande désarticulation entre les enjeux locaux et le système mondial qui s'y superposent.

En effet, la permanence des réseaux illégaux de l'immigration et de la contrebande est alimentée par les écarts croissants de développement entre les deux rives, et ces réseaux ne cessent de se développer malgré les stratégies sécuritaires de barricadement de l'espace européen.

31 Ensuite, la faveur actuelle donnée au développement économique a de nombreux effets pervers. D'un point de vue social, le succès des projets en cours est loin d'être avéré et les voies souhaitables à une inversion des tendances ne semblent pas celles choisies pour le moment. D'un point de vue environnemental, il est certain que les projets en cours se sont complètement affranchis des normes ou des législations qui existent puisque une grande partie des espaces maritimes et terrestres du détroit de Gibraltar sont réglementés par des zonages de protection de niveau régional à européen.

Ces dysfonctionnements amènent alors à la construction d'un espace paradoxal, mais à l'image du monde, où tout est mis en place pour la fluidité et la gestion optimale des circulations marchandes alors que s'opposent barricades, postes de contrôles et législations discriminatoires aux mobilités humaines.

\section{Les premiers pas vers la gestion commune}

Pourtant, le tableau n'est pas complètement sombre sinon il serait illusoire de réfléchir à des perspectives d'intégration.

Le Maroc effectue près des deux tiers de son commerce extérieur avec l'Union Européenne dont $22,1 \%$ avec la France, suivie de l'Espagne (13,2 \%), de l'Italie (5,9\%), de l'Allemagne $(4,2 \%)$ et de la Grande-Bretagne $(3,6 \%)^{4}$. Cette situation lui a valu d'obtenir, en 2008, un statut avancé avec l'U.E dans le cadre de sa politique de voisinage. Il est le premier pays sud-méditerranéen à entrer dans cette catégorie et à renforcer ainsi ses liens politiques et économiques avec l'UE dans l'objectif d'intégrer à terme le marché unique européen. Il est, en particulier, un partenaire politique privilégié de l'Espagne puisqu'ils sont souvent obligés de coopérer en matière de 
gestion des flux migratoires. De 1956 à 2004, soit en 50 ans de relations de voisinage, l'Espagne et le Maroc ont signé 104 accords dont plus d'un tiers concernent le détroit de Gibraltar, les modalités de circulation des personnes et la coopération entre les autorités de frontières (Ramón Remacha, 2006). Cette consolidation des liens eurochérifiens est un préalable à une coopération plus étroite de type intégration frontalière entre le Tangérois et l'Andalousie. Ces régions auraient incontestablement un rôle à jouer dans une politique de voisinage encore plus étroite avec le Maroc, ne serait-ce que dans la réception et la gestion du flux commercial. Une telle situation serait bénéfique à une massification des trafics et à une extension des arrière-pays des ports de Tanger-Med et d'Algeciras.

Enfin, à l'échelle régionale, ce sont les questions environnementales qui ont connu les avancées les plus significatives. En effet, le 25 octobre 2006, l'UNESCO a approuvé le premier projet de Réserve de Biosphère Intercontinentale, proposé par les comités $\mathrm{MaB}$ (Man and Biosphere) de la région Andalousie et du royaume du Maroc. Le territoire proposé, d'une superficie d'un million d'hectares, est déterminé sur la base du large espace de communication entre le continent européen et le continent africain, constitué par le sud de l'Andalousie et le nord du Maroc, englobant donc tout le détroit de Gibraltar et les nombreux parcs naturels, réserves naturelles, monumentos naturales, et sites d'intérêts biologiques et écologiques des deux rives. La gestion commune de tous ces espaces protégées favorise une politique commune de conservation et d'usage des ressources naturelles, premiers pas communs vers une intégration régionale des territoires frontaliers du détroit de Gibraltar.

\section{Conclusion}

Le détroit de Gibraltar est traversé par des flux et des influences multiples, parfois opposés, qui en font un espace original d'observation de la mondialisation et de ses effets sur les territoires. L'espace en question révèle aujourd'hui une offre de transport renouvelée et moderne qui permet une intégration des territoires riverains aux réseaux du transport mondial. Cependant, la faible articulation entre les logiques commerciales d'une part et les intérêts économiques et sociaux locaux d'autre part laisse entrevoir certaines frictions territoriales capables de fragiliser l'espace en construction. S'oppose alors sur ce territoire un espace de la fluidité entretenu par des intérêts extérieurs, celui des flux Est-Ouest, et un espace de la fixité aux réalités multiscalaires et imposé par les difficultés de la relation Nord-Sud (Hesse et Rodrigue, 2004). Les tensions engendrées par la fragilité du territoire invitent alors à une réflexion sur une coopération et une solidarité entre les deux rives afin de dépasser les difficultés liées à la fragmentation spatiale de ce terraqué.

Unir les deux rives d'un détroit par une coopération transfrontalière est un système qui a déjà fait ses preuves depuis 1997 sur les rivages de l'Øresund et qui est à l'étude sur le Pas de Calais (programme européen Interreg IVA France (Manche) - Angleterre). La difficulté de ce genre de projets réside dans le fait que les rivages d'un détroit sont souvent isolés par la coupure d'eau qui s'oppose à leur mise en contact. En recentrant les enjeux sur la gestion de cette coupure d'eau, ils font alors de la mer le support de l'espace en construction. De séparatrice la mer devient intégratrice, animant ainsi le terraqué qu'elle a formé. Sur l'Øresund comme sur le Pas de Calais, les deux rives sont dorénavant unies par un lien fixe. Projet qui existe sur le détroit de Gibraltar depuis 
1869, preuve d'une volonté ancienne de lier les deux rives, et pour lequel des études de faisabilité ont été réalisées au cours des décennies 1980 et 1990. Mais cette union euroafricaine prête encore à controverse, à l'heure d'une Europe de plus en plus protectrice à l'égard de ses frontières extérieures.

\section{BIBLIOGRAPHIE}

Arab C., 2009. Les Aït Ayad. La circulation migratoire des Marocains entre la France, l'Espagne et l'Italie. PUR, Rennes, 358 p.

Bureau International du Travail (BIT), 2003. Emploi et politique sociale dans les zones franches d'exportation. 286e session de la commission de l'emploi et de la politique sociale, Genève, 24 p., http://ilo.org/public/libdoc/ilo/GB/286/GB.286_esp_3_fren.pdf

Bost F., 2007. Les zones franches interface de la mondialisation. Annales de Géographie, $\mathrm{n}^{\circ} 658, \mathrm{p}$ 563-585.

Cherkaoui A., 2006. Le détroit de Gibraltar : tendances actuelles et conjectures. In : Acosta Sánchez M (dir.), Las dimensiones internacionales del Estrecho de Gibraltar. Dykinson S.L, Madrid, p. 335-342.

Coudroy de Lille L., 2007. Les zones économiques spéciales en Pologne : un tremplin pour l'emploi régional ou des miettes de mondialisation? Annales de Géographie, nº 658, p. 645-666.

Ducruet C., 2005. Structures et dynamiques spatiales des villes portuaires du local au global. Mappemonde, $\mathrm{n}^{\circ} 77$ (1-2005), 6 p. http://mappemonde.mgm.fr/num5/articles/art05106.html Charlier J., 1990. Le deuxième souffle du canal de Suez. Transports, nº 341, p. 314-321.

Fremont A., 1996. L'espace maritime et marchand : pour une problématique. L'Espace Géographique, n³ 3. p. 203-213.

Fremont A., 2007. Le monde en boîte. Les Collections de l'INRETS, Paris, 145 p.

Hesse M., Rodrigue J-P., 2004. The transport geography of logistics and freight distribution. Journal of transport geography, vol. 12, n³, p. 171-184.

Guillaume J., 1999. Le canal de Suez à la fin du XX ${ }^{\mathrm{e}}$ siècle. Les Cahiers Nantais, nº 52, p. 35-44.

Guillaume J., 2005. Les routes maritimes, leurs traces et leurs marques. Le monde en réseaux. Lieux visibles, liens invisibles, Festival International de Géographie 30 septembre-2 octobre 2005, St-Dié-des-Vosges, http://archives-fig-st-die.cndp.fr/actes/actes_2005/guillaume/article.htm.

Marcadon J., 2004. Quelques conséquences de l'arrivée des méga porte-conteneurs. Belgeo, $\mathrm{n}^{\circ} 4$, p. 419-431.

Mareï N, 2008. Les enjeux maritimes et portuaires du détroit de Gibraltar. Note de Synthèse $n^{\circ} 105$ de l'ISEMAR, mai 2008

Ministère de l'économie et des finances, 2008. La logistique du commerce au Maroc : état des lieux et pistes de développement. Etudes de la direction des études et des prévisions financières, Rabat, $27 \mathrm{p}$. http://doc.abhatoo.net.ma/doc/IMG/pdf/LOGISTIQUEDUCOMMERCEMAROC.pdf 
Murtland M., 2011. Ferries to Marocco. Ferry and Cruise Annual 2011. Ferry Publications, Ramsey, p. $90-97$.

Offner J-M., 1993. Les effets structurants du transport : mythe politique, mystification scientifique. L'Espace Géographique, $\mathrm{n}^{\circ}$ 3, p. 233-242.

Ramon Remacha J., 2006. Le détroit de Gibraltar comme zone frontière. In : Acosta Sánchez M. (dir.), Las dimensiones internacionales del Estrecho de Gibraltar. Dykinson S.L, Madrid, p. 325-334.

Tarrius A., 1992. Les fourmis d'Europe. Migrants riches, migrants pauvres et nouvelles villes internationales. L'Harmattan, Paris, 207 p.

Vigarié A., 1995. La mer et la géostratégie des nations, Paris, Economica, 432 p.

Zurlo Y., 2006. Ceuta et Melilla. Histoire, représentations et devenir de deux enclaves espagnoles.

L'Harmattan, Paris. 317 p.

\section{NOTES}

1. L'ensemble des trafics portuaires sont données par les autorités portuaires. Il existe des chiffres plus récents que ceux de l'année 2007 mais ils ne reflètent pas aussi bien les tendances étudiées car ils sont parfois fortement impactés par la crise.

2. Isemar / Le Marin, 6-06-08.

3. A titre indicatif, 13 millions de passagers ont traversé le détroit du Pas de Calais en 2010 (doverport.co.uk), 6 millions le détroit de Malacca (singaporecruise.com.sg) et 8 millions le détroit de l'øresund (tendensoresund.org).

4. Le Marin, 4-05-07.

\section{RÉSUMÉS}

Le détroit de Gibraltar est une voie de passage intercontinentale et interocéanique majeure. Tout autour du détroit trois Etats ont pris place: l'Espagne, le Maroc, le Royaume-Uni. Ils sont imbriquées les uns aux autres par un jeu d'enclaves territoriales et dépendent les uns des autres pour la circulation des biens et des personnes à travers le détroit. Cette circulation, intégrée dans les grands flux marchands et humains qui dominent la planète, fait toute l'attractivité des lieux et entraîne une organisation maritime et terrestre singulière autour de ce seuil océanique. En particulier, les terminaux portuaires se sont développés de manière spectaculaire et accueillent aujourd'hui les principaux transporteurs mondiaux. Cette faveur donnée au développement économique a de nombreux effets pervers, qui s'ils ne sont pas pris en compte rapidement, mèneront à une fragilisation des territoires frontaliers du détroit.

The Strait of Gibraltar is a major intercontinental and interoceanic route for maritime traffic. Three states surrend the Strait: Spain, Morocco, and the United Kingdom. Being territorial enclaves, they overlap and depend of each others for regulating the circulation of goods and people through the strait. This intercirculation, integrated in major merchant and human flows which dominate the world, makes all the interest of the place and creates a singular maritime 
and spatial organization around this oceanic chokepoint. In particular, port terminals have grown rapidly and hosting major global carriers. But, the preminence given to the economic development has many perverse effects on the territories bordering the strait.

\section{INDEX}

Mots-clés : circulation internationale, détroit international, intégration régionale, mondialisation, port, transport maritime

Keywords : globalisation, harbour, international circulation, international strait, maritime transport, regional integration

Thèmes : Sur le Champ - Sur le Terrain

\section{AUTEUR}

\section{NORA MAREİ}

Nora Mareï marei.nora@gmail.com est membre du Laboratoire Géolittomer, LETG UMR 6554 CNRS et ATER à l'Université de Nantes. Elle a publié :

- Mareï N., 2009. Le détroit de Gibraltar fenêtre sur le monde pour l'Espagne. Historiens et Géographes, $n^{\circ}$ 408. Numéro spécial, L’Espagne, les métamorphoses d'une puissance économique sous la coord. de N. Baron Yellès.

- Mareï N., Tourret P., 2009. Les industries maritimes, les territoires et leur organisation. Cahiers Nantais, $\mathrm{n}^{\circ}$ 1-2009, p 5-17.

- Mareï N., 2008. Les enjeux maritimes et portuaires du détroit de Gibraltar. Note de Synthèse $\mathrm{n}^{\circ} 105$ de l'ISEMAR, mai 2008, 4 p. ; reprise : Annales 2008 de l'Institut Méditerranéen des Transports Maritimes, dossier « vers un réseau de plates-formes logistiques en Méditerranée : mythe ou réalité ? ", p. 125-133; reprise : Le Marin $\mathrm{n}^{\circ}$ 3174, 9 mai 2008. 